RICYDE. Revista Internacional de Ciencias del Deporte doi: $10.5232 /$ ricyde

Rev. int. cienc. deporte

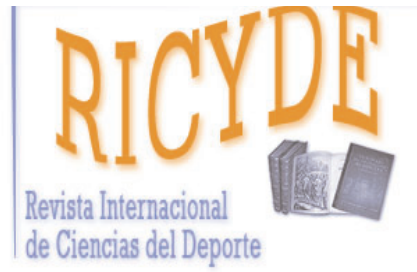

RICYDE. Revista Internacional de Ciencias del Deporte VOLUMEN XI - AÑO XI

páginas:226-244 ISSN : 1885-3137 No 41 - Julio - 2015

\title{
Análisis del ataque posicional de balonmano playa masculino y femenino mediante coordenadas polares Analysis of positional attack in beach handball male and female with polar coordinates
}

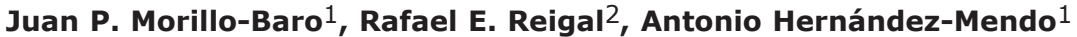

1. Universidad de Málaga. España

2. Universidad de Granada. España

\section{Resumen}

La presente investigación tiene como objetivo aportar una perspectiva novedosa en la comprensión y diferenciación de las conductas de juego en la fase de ataque posicional en el balonmano playa masculino y femenino. Para ello se analizaron 28 partidos de alto nivel con el programa informático Hoisan. Se utilizó un diseño Observacional de carácter nomotético, de seguimiento y multidimensional con un sistema taxonómico metodológicamente validado. Los datos fueron sometidos a un análisis de coordenadas polares en su versión genuina. Para llevar a cabo estos análisis se escogieron siete conductas focales relativas, principalmente, a los jugadores que finalizan el ataque y el modo de realizarlo. Los resultados mostraron diferencias entre las conductas de apareo en la categoría masculina y femenina. Destaca que el ataque posicional en la categoría femenina se orienta hacia zonas de finalización izquierdas ante un sistema defensivo abierto y depende más de la jugadora que adquiere el rol de doble portera (especialista) que en la categoría masculina, donde las responsabilidades están más repartidas y el ataque se dirige hacia la banda derecha ante un sistema defensivo cerrado. El lanzamiento en giro se ha mostrado como el principal recurso ofensivo en ambas categorías.

Palabras claves: metodología observacional; coordenadas polares; interacción; balonmano playa.

\begin{abstract}
This research aims to provide a new perspective on understanding and differentiation of play behavior in the phase of positional attack in the male and female beach handball. 28 high-level games with Hoisan software were analyzed. The observational design used is nomothetic, monitoring and multidimensional. The taxonomic system has been validated methodologically. Data were subjected to analysis of polar coordinates in its genuine version. To carry out these analyzes on seven focal behaviors were chosen mainly for players who complete the attack and how to create it. The results showed differences in mating behavior in the male and female category. Emphasizes that the positional attack in the female category is geared towards areas left defensive end to an open system depends more on the player who takes the role of caretaker double (specialist) in the men's category, where responsibilities are more spread and attack directed toward the right sideline before a closed defense system. The shoot in pirouette has been shown as the main offensive action in both categories.
\end{abstract}

Key words: observational methodology; polar coordinates; interaction; beach handball. 
Morillo-Baro, J. P.; Reigal, R.; L.; Hernández-Mendo, A. (2015). Análisis del ataque posicional de balonmano playa masculino y femenino mediante coordenadas polares. RICYDE. Revista internacional de ciencias del deporte, 41(11), 226-244. http://dx.doi.org/10.5232/ricyde2015.04103

\section{Introducción}

$\mathrm{D}$ esde su origen, a principios de los años noventa, el balonmano playa como modalidad de juego del balonmano ha ido evolucionando en todas las áreas (técnica, táctica, reglas del juego, etc.) hasta convertirse en una realidad deportiva presente en los cinco continentes y reglada por las más altas instituciones deportivas. La apuesta de la Federación Internacional de Balonmano (IHF) y la Federación Europea de Balonmano (EHF) por esta modalidad es evidente. Así, por ejemplo, se disputan con regularidad campeonatos del mundo y campeonatos continentales.

Esta modalidad presenta diferencias notables con las reglas de juego del balonmano pista que lo han convertido en un deporte nuevo. La normativa es distinta, destacando, por ejemplo, la diferente puntuación de los goles dependiendo de la manera en que se consigan. Esta particularidad ha permitido, por un lado, centrar la unidad de análisis en el ataque posicional; considerando éste desde la realización del cambio defensa ataque del portero hasta el cambio de posesión del móvil. Aspecto que ha favorecido obtener la fiabilidad necesaria en el proceso de calidad del dato en la construcción de la herramienta de observación. Y por otro, centrar la investigación del análisis de coordenadas polares en las conductas focales que son características de este deporte, como las asistencias y finalización del doble portero y los lanzamientos en flight o en giro.

Efectivamente, y coincidiendo con Carling, Williams, y Reilly (2005) el rendimiento del juego deportivo colectivo es mucho más difícil de evaluar que el de los deportes individuales; y el balonmano playa, al igual que otros deportes colectivos, se caracteriza por tener una gran complejidad de comportamientos que dificultan su observación y análisis. Para los entrenadores es necesario investigar y obtener información científica sobre las demandas reales en competición con el objetivo de optimizar los programas de entrenamiento desde un punto de vista técnico-táctico (Matsushigue, Hartmann, y Franchini, 2009; Tornello, Capranica, Chiodo, Minganti, y Tessitore, 2013; Tornello, Capranica, Minganti, Chiodo, Condello, y Tessitore, 2014). Habitualmente se utilizan estudios que analizan sólo la frecuencia de ocurrencia de los eventos por lo cual no llegan a analizar un aspecto clave en el juego, como es su dimensión temporal (Sarmiento, Marques, Martins, Anguera, Campaniço, y Leitão, 2011). Ciertamente, en los últimos años se ha incrementado el número de investigaciones sobre las acciones de juego en deportes colectivos basadas en la Metodología Observacional (e.g., Anguera y Hernández-Mendo, 2013, 2014; Casamichana, Castellano, Blanco-Villaseñor, y Usabiaga, 2012; Garzón, Lapresa, Anguera, y Arana, 2011; González, Botejara, Puñales, Trejo, y Ruy, 2013; Sousa, Prudente, Sequeira, y Hernández-Mendo, 2014) y el análisis de coordenadas polares junto con el secuencial (técnica de retardos y markoviano), característicos de esta metodología (Anguera y Hernández-Mendo, 2013, 2014), utiliza el parámetro orden en su ejecución.

El análisis de coordenadas polares es una herramienta de gran utilidad, ya que se trata de "una técnica analítica sumamente potente que incide de lleno en el análisis de la complejidad" (Martín y Lago, 2005, p. 143). La utilización de esta técnica permite reducir la acumulación de información a unos pocos parámetros vectoriales que se materializan en un mapa interrelacional que posibilita conjugar las perspectivas retrospectiva y prospectiva (Gorospe y Anguera, 2000). Este mapa de relaciones vincula una determinada categoría (conducta focal o criterio) con todas las propias del sistema. Se trata de una representación vectorial donde el ángulo del vector indica la naturaleza de la relación y su radio o módulo la intensidad de la misma (Anguera, Blanco, y Losada, 1997). 
Morillo-Baro, J. P.; Reigal, R.; L.; Hernández-Mendo, A. (2015). Análisis del ataque posicional de balonmano playa masculino y femenino mediante coordenadas polares. RICYDE. Revista internacional de ciencias del deporte, 41(11), 226-244. http://dx.doi.org/10.5232/ricyde2015.04103

Este tipo de técnica ha cobrado una gran relevancia dentro del mundo del deporte, llevándose a cabo en diversas disciplinas como el tenis, el fútbol o el balonmano (Castellano y Hernández-Mendo, 2003; Castellano, Hernández-Mendo, Morales-Sánchez, y Anguera, 2007; González y col., 2013; Hernández-Mendo y Anguera, 1998; Perea, 2008; Perea, Castellano, Alday, y Hernández-Mendo, 2012). Así, Gorospe y Anguera (2000) concluyeron aplicándola al tenis que, considerando los retardos negativos (retrospectividad propia) en la técnica genuina, versión mejorada de la inicial planteada por Sackett (1980), genera una red completa de interrelaciones entre categorías a partir de una conducta criterio. En fútbol, por ejemplo, Hernández-Mendo y Anguera (1999) aplican este análisis a un sistema de categorías utilizado para el estudio de partidos de primera división. Y, más recientemente, Robles y Castellano (2012) valoraron las diferencias en el modelo de juego de la selección española de fútbol y sus rivales en la Eurocopa 2008 y Mundial 2010. Prudente, Garganta, y Anguera (2010) destacan las posibilidades que brinda el análisis secuencial y las coordenadas polares para estudiar las conductas del juego en balonmano. Por su parte, González y col. (2013) analizaron el Campeonato del mundo 2011 y JJOO 2012, estudiando la eficacia de la finalización del ataque en los momentos finales de partidos igualados de balonmano; destacando la vinculación entre la interceptación y la obtención de gol tanto en los equipos ganadores como en los perdedores, la asociación de lanzamientos atajados a ganadores y la relación de fallos de lanzamiento y errores previos al lanzamiento en perdedores.

Considerando los antecedentes del balonmano playa como disciplina deportiva objeto de estudio científico, el objetivo de esta investigación fue identificar mediante análisis de coordenadas polares las relaciones y asociaciones entre las diferentes conductas que suceden en el ataque posicional del balonmano playa, diferenciándolo en función de las categorías masculina o femenina. Para ello, se utilizó la técnica de las coordenadas polares.

\section{Método}

En este estudio se ha usado un diseño situado en el cuarto cuadrante de carácter seguimiento/nomotético/multidimensional (Anguera, Blanco-Villaseñor, Hernández-Mendo, y Losada, 2011).

\section{Participantes}

Tras un análisis de generalizabilidad se observaron un total de 28 partidos completos del máximo nivel. La aplicación de esta teoría permite estimar el grado de generalización de un diseño de medida con respecto a las condiciones particulares de un valor teórico buscado. El coeficiente de generalizabilidad permite estimar el ajuste de la media observada a la media de todas las observaciones posibles (Blanco, Castellano, y Hernández-Mendo, 2000; BlancoVillaseñor, Castellano, Hernández-Mendo, Sánchez-López, y Usabiaga, 2014).

Partidos analizados en categoría masculina:

1. Selección nacional absoluta masculina en su participación en el campeonato de Europa de 2013: España-Dinamarca, España-Noruega, España-Polonia, España-Suiza y España-Ucrania.

2. Selección nacional sub'19 masculina en su participación en el campeonato de Europa de 2013: España-Dinamarca, España-Rusia, España-Serbia, España-Suiza y EspañaSerbia (cuartos de final).

3. Clubes masculinos durante el campeonato de España de 2013: C. BMP. AlcaláGuarapo Almería, ARHS-C. BMP. Ciudad de Málaga, C. BMP. Barbate-C. BMP. Algeciras, C. BMP. Ciudad de Málaga-C. BMP. Barbate y C. BMP. Barbate-C. BMP. Algeciras (semifinales). 
Morillo-Baro, J. P.; Reigal, R.; L.; Hernández-Mendo, A. (2015). Análisis del ataque posicional de balonmano playa masculino y femenino mediante coordenadas polares. RICYDE. Revista internacional de ciencias del deporte, 41(11), 226-244. http://dx.doi.org/10.5232/ricyde2015.04103

Partidos analizados en categoría femenina:

1. Selección nacional absoluta femenina en su participación en el campeonato de Europa de 2013: España-Turquía, España-Noruega, España-Hungría, España-Italia y EspañaRusia.

2. Selección nacional sub'19 femenina en su participación en el campeonato de Europa de 2013: España-Dinamarca, España-Rusia, España-Noruega.

3. Clubes femeninos durante el campeonato de España de 2013: C. Ciudad de Málaga Mijas- C. BMP. Getafe, Cubas Llopis Sevilla-C. BMP. Getafe, Deporte y Empresa Málaga- Vícar Goya, Jugui SOS-Vícar Goya y Cubas Llopis Sevilla-C. BMP. Getafe (final).

\section{Instrumentos}

Se ha utilizado el programa informático Hoisan (Hernández-Mendo, López-López, Castellano, Morales-Sánchez, y Pastrana, 2012; Hernández-Mendo y col., 2014) para realizar la codificación del flujo comportamental ya que este programa calcula, además, el análisis de coordenadas polares y su representación vectorial.

La herramienta de observación, diseñada $a d$ hoc, ha superado las pruebas de calidad del dato exigibles en metodología observacional. Desde un punto de vista cuantitativo, los resultados de los coeficientes de correlación Tau b de Kendall, Pearson y Spearman muestran unos índices mínimos de 0.993, los índices Kappa de Cohen calculados para cada criterio de la herramienta se sitúan en un mínimo de 0.917 y los índices de generalizabilidad son óptimos. Apoyada en el carácter cualitativo, se ha utilizado la concordancia consensuada (Anguera, 1990) para lograr el acuerdo entre los observadores en la elaboración del sistema de codificación, la confección del protocolo de observación y el entrenamiento minucioso de los mismos (Morillo y Hernández-Mendo, 2015).

En la tabla 1, se observan los valores de los coeficientes de correlación realizados; y en la tabla 2, los correspondientes a los índices de Kappa de Cohen (Morillo y Hernández-Mendo, 2015).

Tabla 1. Valores de los coeficientes de correlación Tau b de Kendall, Pearson y Spearman.

\begin{tabular}{|c|c|c|}
\hline \multicolumn{3}{|c|}{ Coeficientes de correlación } \\
\hline $\begin{array}{c}\text { Coeficiente } \\
\text { Sesión entera }\end{array}$ & $\begin{array}{l}\text { Concordancia intra } \\
\text { (Obs. } 1 \text { vs Obs. 1bis) }\end{array}$ & $\begin{array}{c}\text { Concordancia inter } \\
\text { (Obs. } 1 \text { vs Obs. 2) }\end{array}$ \\
\hline Tau b de Kendall & 0.998 & 0.993 \\
\hline Pearson & 0.999 & 0.998 \\
\hline Spearman & 1.000 & 0.999 \\
\hline
\end{tabular}

Tabla 2. Valores de los índices de kappa de Cohen en relación a los criterios y a la sesión completa.

\begin{tabular}{|c|c|c|}
\hline \multicolumn{3}{|c|}{ Índices de Kappa de Cohen } \\
\hline $\begin{array}{c}\text { Grupo de conductas } \\
\text { (criterios) }\end{array}$ & $\begin{array}{l}\text { Concordancia intra } \\
\text { (Obs.1 vs Obs.1Bis) }\end{array}$ & $\begin{array}{c}\text { Concordancia inter } \\
\text { (Obs.1 vs Obs.2) }\end{array}$ \\
\hline Minuto número & 1.000 & 1.000 \\
\hline Marcador & 1.000 & 1.000 \\
\hline Equilibrio numérico & 1.000 & 1.000 \\
\hline Sistema defensivo & 1.000 & 0.917 \\
\hline Zona finalización & 1.000 & 0.958 \\
\hline Banda cambios & 1.000 & 1.000 \\
\hline Jugador asiste & 0.955 & 0.955 \\
\hline Jugador finaliza & 1.000 & 1.000 \\
\hline Modo finalización & 1.000 & 1.000 \\
\hline Resultado finalización & 1.000 & 1.000 \\
\hline
\end{tabular}


Morillo-Baro, J. P.; Reigal, R.; L.; Hernández-Mendo, A. (2015). Análisis del ataque posicional de balonmano playa masculino y femenino mediante coordenadas polares. RICYDE. Revista internacional de ciencias del deporte, 41(11), 226-244. http://dx.doi.org/10.5232/ricyde2015.04103

\begin{tabular}{|l|c|c|}
\hline \multicolumn{3}{|c|}{ Índices de Kappa de Cohen } \\
\hline $\begin{array}{c}\text { Grupo de conductas } \\
\text { (criterios) }\end{array}$ & $\begin{array}{c}\text { Concordancia intra } \\
\text { (Obs.1 vs Obs.1Bis) }\end{array}$ & $\begin{array}{c}\text { Concordancia inter } \\
\text { (Obs.1 vs Obs.2) }\end{array}$ \\
\hline Duración & 0.936 & 0.936 \\
\hline Sesión completa & 0.987 & 0.981 \\
\hline
\end{tabular}

La herramienta está formada por 11 criterios y 90 categorías que se corresponden con el desarrollo cronológico de un ataque (Morillo y Hernández-Mendo, 2015).

Tabla 3. Listado de categorías correspondientes a cada criterio y sistema de codificación.

\begin{tabular}{|c|c|}
\hline Criterios & Categorías \\
\hline Minuto número & $\begin{array}{l}\text { M1: minuto uno del primer set. } \\
\text { M2: minuto dos del primer set. } \\
\text { M3: minuto tres del primer set. } \\
\text { M4: minuto cuatro del primer set. } \\
\text { M5: minuto cinco del primer set. } \\
\text { M6: minuto seis del primer set. } \\
\text { M7: minuto siete del primer set. } \\
\text { M8: minuto ocho del primer set. } \\
\text { M9: minuto nueve del primer set. } \\
\text { M10: minuto diez del primer set. } \\
\text { M11: minuto uno del segundo set. } \\
\text { M12: minuto dos del segundo set. } \\
\text { M13: minuto tres del segundo set. } \\
\text { M14: minuto cuatro del segundo set. } \\
\text { M15: minuto cinco del segundo set. } \\
\text { M16: minuto seis del segundo set. } \\
\text { M17: minuto siete del segundo set. } \\
\text { M18: minuto ocho del segundo set. } \\
\text { M19: minuto nueve del segundo set. } \\
\text { M20: minuto diez del segundo set. } \\
\text { MGO1: gol de oro del primer set. } \\
\text { MGO2: gol de oro del segundo set. }\end{array}$ \\
\hline Marcador & $\begin{array}{l}\text { MPATE: empate. } \\
\text { 1FAV: ganando de uno el equipo observado. } \\
\text { 2FAV: ganando de dos el equipo observado. } \\
\text { M2FAV: ganando de más de dos el equipo observado. } \\
\text { 1CON: perdiendo de uno el equipo observado. } \\
\text { 2CON: perdiendo de dos el equipo observado. } \\
\text { M2CON: perdiendo de más de dos el equipo observado. }\end{array}$ \\
\hline Equilibrio numérico & $\begin{array}{l}\text { IGUAL: igualdad. } \\
\text { 1SUP: uno en superioridad el equipo observado. } \\
\text { M1SUP: más de uno en superioridad el equipo observado. } \\
\text { 1INF: uno en inferioridad el equipo observado. } \\
\text { M1INF: más de uno en inferioridad el equipo observado. }\end{array}$ \\
\hline Sistema defensivo & $\begin{array}{l}\text { S30: Sistema defensivo } 3: 0 . \\
\text { S21: Sistema defensivo } 2: 1 . \\
\text { S2M1: Sistema defensivo } 2+1 \\
\text { SPRES: Sistema defensivo individual. } \\
\text { SREPL: Sistema defensivo repliegue. } \\
\text { S20: Sistema defensivo 2:0. }\end{array}$ \\
\hline Zona finalización & $\begin{array}{l}\text { Z1: finalización en la zona } 1 . \\
\text { Z2: finalización en la zona } 2 \text {. } \\
\text { Z3: finalización en la zona } 3 \text {. } \\
\text { Z4: finalización en la zona } 4 . \\
\text { Z5: finalización en la zona } 5 \text {. } \\
\text { Z6: finalización en la zona } 6 \text {. } \\
\text { Z7: finalización en la zona } 7 \text {. }\end{array}$ \\
\hline
\end{tabular}


Morillo-Baro, J. P.; Reigal, R.; L.; Hernández-Mendo, A. (2015). Análisis del ataque posicional de balonmano playa masculino y femenino mediante coordenadas polares. RICYDE. Revista internacional de ciencias del deporte, 41(11), 226-244. http://dx.doi.org/10.5232/ricyde2015.04103

\begin{tabular}{|c|c|}
\hline Criterios & Categorías \\
\hline & $\begin{array}{l}\text { Z8: finalización en la zona } 8 \text {. } \\
\text { Z9: finalización en la zona } 9 \text {. }\end{array}$ \\
\hline Banda cambios & $\begin{array}{l}\text { BCSI: finalización en una de las zonas colindantes con la banda de } \\
\text { cambios del equipo observado. } \\
\text { BCNO: finalización en una de las zonas colindantes con la banda de } \\
\text { cambios del equipo no observado. } \\
\text { BCMED: finalización en una de las zonas centrales. }\end{array}$ \\
\hline Jugador asiste & $\begin{array}{l}\text { APETO: asiste el doble portero. } \\
\text { AXTRI: asiste el lateral izquierdo. } \\
\text { AXTRD: asiste el lateral derecho. } \\
\text { ACENT: asiste el central. } \\
\text { APIV: asiste el pivote. } \\
\text { NASIS: nadie asiste. }\end{array}$ \\
\hline Jugador finaliza & $\begin{array}{l}\text { FPETO: finaliza el doble portero. } \\
\text { FXTRI: finaliza el lateral izquierdo. } \\
\text { FXTRD: finaliza el lateral derecho. } \\
\text { FCENT: finaliza el central. } \\
\text { FPIV: finaliza el pivote. }\end{array}$ \\
\hline Modo finalización & $\begin{array}{l}\text { FLY: finalización en lanzamiento en flight. } \\
\text { GIRO: finalización en lanzamiento en giro. } \\
\text { LANZ: finalización en lanzamiento. } \\
\text { 6M: finalización en lanzamiento de } 6 \text { metros. } \\
\text { ERRPR: finalización en error de pase o recepción. } \\
\text { FTECN: finalización en falta técnica. }\end{array}$ \\
\hline Resultado finalización & $\begin{array}{l}\text { GOL1: gol de valor simple. } \\
\text { GOL2: gol de valor doble. } \\
\text { ERLAN: error de lanzamiento. } \\
\text { CPOSE: cambio de posesión. } \\
\text { G1SA: gol de valor simple y sanción. } \\
\text { G2SA: gol de valor doble y sanción. } \\
\text { 6MGOL: gol de lanzamiento de } 6 \text { metros. } \\
\text { 6MFA: error de lanzamiento de } 6 \text { metros. } \\
\text { GOL1R: gol de valor simple de rebote. } \\
\text { GOL2R: gol de valor doble de rebote. } \\
\text { 6MGSA: gol de lanzamiento de } 6 \text { metros y sanción. } \\
\text { 6MFSA: error de lanzamiento de } 6 \text { metros y sanción. } \\
\text { G1RSA: gol de valor simple de rebote y sanción. } \\
\text { G2RSA: gol de valor doble de rebote y sanción. }\end{array}$ \\
\hline Duración & $\begin{array}{l}\text { D05: entre } 0 \text { y } 5 \text { segundos. } \\
\text { D610: entre } 6 \text { y } 10 \text { segundos. } \\
\text { D1115: entre } 11 \text { y } 15 \text { segundos. } \\
\text { D1620: entre } 16 \text { y } 20 \text { segundos. } \\
\text { D2125: entre } 21 \text { y } 25 \text { segundos. } \\
\text { D2630: entre } 26 \text { y } 30 \text { segundos. } \\
\text { DM30: más de } 30 \text { segundos. }\end{array}$ \\
\hline
\end{tabular}

Las categorías focales utilizadas para realizar el análisis de coordenadas polares han sido siete:

1. APETO: Asiste el doble portero.

2. ERRPR: Finalización en error de pase o recepción.

3. FLY: Finalización en lanzamiento en flight.

4. FPETO: Finaliza el doble portero.

5. FPIV: Finaliza el pivote.

6. GIRO: Finalización en lanzamiento en giro.

7. LANZ: Finalización en lanzamiento. 
Morillo-Baro, J. P.; Reigal, R.; L.; Hernández-Mendo, A. (2015). Análisis del ataque posicional de balonmano playa masculino y femenino mediante coordenadas polares. RICYDE. Revista internacional de ciencias del deporte, 41(11), 226-244. http://dx.doi.org/10.5232/ricyde2015.04103

Las conductas de apareo están formadas por el resto de categorías de la herramienta exceptuando las pertenecientes al criterio "Minuto número".

\section{Procedimiento}

La codificación y posterior análisis de coordenadas polares se realizó mediante el programa informático Hoisan (Hernández-Mendo et al., 2012, 2014). El mapa de coordenadas polares que ofrece esta técnica, parte de los valores Zsum y del número de los retardos considerados (en este caso 5) para la perspectiva prospectiva y $(-5)$ para la retrospectiva, que permitirá establecer la existencia de dependencia excitatoria o inhibitoria (según sean los valores positivos o negativos, respectivamente) entre la conducta focal y las conductas condicionadas (Santoyo y Anguera, 1993). Estas últimas equivalen a una imagen especular de la última, penúltima, etc. conductas anteriores a la conducta criterio, o primera, segunda, tercera, etc. posteriores a la conducta criterio, dándose entre todas ellas una relación estable $\mathrm{y}$, desvelándose como "preparatorias" o "resultantes" a la ocurrencia de la conducta criterio (Gorospe y Anguera, 2000).

En primer lugar se realizó un análisis secuencial para cada categoría (masculina y femenina) de todas las observaciones realizadas conjuntamente con cada una de las siete categorías focales seleccionadas, obteniendo los Residuos Ajustados $(z)$ del rango de retardos $(-5,5)$. A partir de estos valores, y para cada conducta focal, se realizaron los cálculos para determinar los parámetros Zsum (prospectivo y retrospectivo), la asignación de cuadrante, el módulo, el ángulo, y el ángulo transformado (AT) para el resto de categorías (Castellano y HernándezMendo, 2003).

Finalmente se obtiene la representación gráfica de coordenadas polares con restrospectividad genuina (Gorospe y Anguera, 2000; Lago y Anguera, 2002). Se consideran relaciones significativas $(p<0.05)$ aquellas en las que el radio o módulo del vector $\geq 1,96$. La caracterización de cada cuadrante es la siguiente (Hernández-Mendo y Anguera, 1999):

Cuadrante I $[+,+]$ : conducta criterio y conducta de apareo mutuamente excitatorias.

Cuadrante II $[-,+]$ : conducta criterio inhibitoria y conducta de apareo excitatoria.

Cuadrante III [-,-]: conducta criterio y conducta de apareo mutuamente inhibitorias.

Cuadrante IV [+,-]: conducta criterio excitatoria y conducta de apareo inhibitoria.

\section{Resultados}

A continuación, y para cada una de las categorías (masculina y femenina), se muestran los mapas conductuales estimados a partir de las categorías focales para cada uno de los cuadrantes.

En la tabla 4 se observa las asociaciones significativas entre cada conducta focal y las conductas de apareo, que, al estar situadas en el cuadrante I, son mutuamente excitatorias. 
Morillo-Baro, J. P.; Reigal, R.; L.; Hernández-Mendo, A. (2015). Análisis del ataque posicional de balonmano playa masculino y femenino mediante coordenadas polares. RICYDE. Revista internacional de ciencias del deporte, 41(11), 226-244. http://dx.doi.org/10.5232/ricyde2015.04103

Tabla 4. Relaciones significativas en el cuadrante I entre las conductas criterio y las conductas de apareo en las dos categorías.

\begin{tabular}{|c|c|c|c|c|c|c|}
\hline Cuadrante I & \multicolumn{3}{|c|}{ Masculino } & \multicolumn{3}{|c|}{ Femenino } \\
\hline $\begin{array}{l}\text { Conducta } \\
\text { criterio }\end{array}$ & $\begin{array}{l}\text { Conducta } \\
\text { de apareo }\end{array}$ & $\begin{array}{l}\text { Módulo } \\
\text { Vector }\end{array}$ & $\begin{array}{l}\text { Ángulo } \\
\text { transformado }\end{array}$ & $\begin{array}{l}\text { Conducta } \\
\text { de apareo }\end{array}$ & $\begin{array}{l}\text { Módulo } \\
\text { Vector }\end{array}$ & $\begin{array}{l}\text { Ángulo } \\
\text { transformado }\end{array}$ \\
\hline \multirow{8}{*}{ APETO } & MPATE & 2.47 & 68.80 & $2 \mathrm{CON}$ & 3.95 & 11.63 \\
\hline & $2 \mathrm{CON}$ & 4.07 & 83.69 & SREPL & 2.40 & 87.70 \\
\hline & S30 & 2.22 & 10.45 & S21 & 3.41 & 52.44 \\
\hline & ZF5 & 2.85 & 71.24 & ZF7 & 2.67 & 39.49 \\
\hline & ZF9 & 5.88 & 54.16 & APETO & 3.97 & 45 \\
\hline & APETO & 3.33 & 45 & FXTRI & 2.11 & 46.26 \\
\hline & FPETO & 2.23 & 84.06 & FLY & 4.50 & 39.59 \\
\hline & FXTRD & 2.77 & 32.44 & ERLAN & 2.28 & 7.72 \\
\hline \multirow{6}{*}{ ERRPR } & S30 & 3.92 & 48.30 & 1FAV & 3.11 & 85.16 \\
\hline & ZF6 & 2.12 & 37.24 & M2FAV & 2.75 & 31.18 \\
\hline & $\mathrm{BCNO}$ & 2.35 & 42 & AXTRI & 3.21 & 3.09 \\
\hline & FXTRD & 2.77 & 52.81 & FPIV & 2.02 & 14.99 \\
\hline & D610 & 4.25 & 45 & FTECN & 2.01 & 8.90 \\
\hline & & & & CPOSE & 2.51 & 23.10 \\
\hline \multirow{7}{*}{ FLY } & $\mathrm{M} 2 \mathrm{CON}$ & 2.46 & 76.02 & ZF4 & 2.13 & 58.29 \\
\hline & S21 & 2.20 & 86.29 & ZF7 & 2.14 & 79.73 \\
\hline & ZF9 & 2.57 & 31.37 & APETO & 4.50 & 50.41 \\
\hline & ACENT & 2.12 & 79.64 & FXTRI & 2.80 & 47.75 \\
\hline & FCENT & 2.09 & 56.36 & & & \\
\hline & LANZ & 2.61 & 70.96 & & & \\
\hline & D610 & 4.61 & 87.94 & & & \\
\hline \multirow{8}{*}{ FPETO } & M2FAV & 2.97 & 80.71 & M2FAV & 2.64 & 7.31 \\
\hline & $\mathrm{S} 30$ & 2.84 & 71.82 & $\mathrm{~S} 30$ & 2.29 & 45.58 \\
\hline & ZF7 & 2.28 & 78.15 & ZF7 & 2.27 & 50.46 \\
\hline & APETO & 2.23 & 5.94 & BCMED & 2.21 & 53.60 \\
\hline & APIV & 2.30 & 10.50 & LANZ & 2.89 & 56.63 \\
\hline & FTECN & 1.99 & 38.11 & & & \\
\hline & CPOSE & 2.35 & 57.13 & & & \\
\hline & D05 & 2.78 & 17.32 & & & \\
\hline \multirow{5}{*}{ FPIV } & M2FAV & 4.33 & 21.48 & $1 \mathrm{CON}$ & 2.84 & 12.51 \\
\hline & BCMED & 1.96 & 52.48 & SPRES & 1.98 & 32.45 \\
\hline & FCENT & 2.13 & 65.50 & ERRPR & 2.02 & 75.01 \\
\hline & GOL1 & 1.99 & 55.83 & D05 & 2.43 & 51.80 \\
\hline & GOL2 & 2.16 & 73.79 & & & \\
\hline \multirow{9}{*}{ GIRO } & $2 \mathrm{FAV}$ & 2.46 & 53.95 & $\mathrm{M} 2 \mathrm{CON}$ & 3.61 & 25.86 \\
\hline & $1 \mathrm{CON}$ & 2 & 77.71 & ZF6 & 4.94 & 39.75 \\
\hline & $\mathrm{M} 2 \mathrm{CON}$ & 3.01 & 59.90 & $\mathrm{BCNO}$ & 2.81 & 35.61 \\
\hline & 1SUP & 1.98 & 0.52 & ACENT & 3.65 & 46.18 \\
\hline & S21 & 2.82 & 52.57 & FXTRD & 5.79 & 37.07 \\
\hline & S20 & 1.98 & 0.52 & GIRO & 6.21 & 45 \\
\hline & GIRO & 3.72 & 45 & GOL2 & 2.69 & 77.97 \\
\hline & D1115 & 3.52 & 35.70 & D1620 & 3.76 & 32.13 \\
\hline & D2630 & 2.57 & 77.03 & & & \\
\hline \multirow{5}{*}{ LANZ } & M2FAV & 7.20 & 83.06 & M2FAV & 2.97 & 32.05 \\
\hline & $\mathrm{S} 30$ & 4.19 & 86.44 & ZF5 & 3 & 56.40 \\
\hline & ZF8 & 2.08 & 34.50 & BCMED & 3.38 & 53.48 \\
\hline & APIV & 2.21 & 54.50 & FPETO & 2.89 & 33.37 \\
\hline & FLY & 2.61 & 19.04 & LANZ & 2.68 & 45 \\
\hline
\end{tabular}

Valor de significación de las relaciones a nivel de $\mathrm{p}<0.05$

Cuando la conducta criterio es APETO (asiste el doble portero) el número de conductas de apareo es el mismo en categoría masculina y femenina (8), aunque sólo coinciden dos. Todas 
Morillo-Baro, J. P.; Reigal, R.; L.; Hernández-Mendo, A. (2015). Análisis del ataque posicional de balonmano playa masculino y femenino mediante coordenadas polares. RICYDE. Revista internacional de ciencias del deporte, 41(11), 226-244. http://dx.doi.org/10.5232/ricyde2015.04103

ellas son asociaciones significativas que presentan mutua excitación de carácter prospectivo y retrospectivo.

En la figura 1, y a modo de ejemplo, se aprecia de forma gráfica uno de los resultados del análisis donde no se encuentran coincidencias en las conductas de apareo entre las dos categorías.

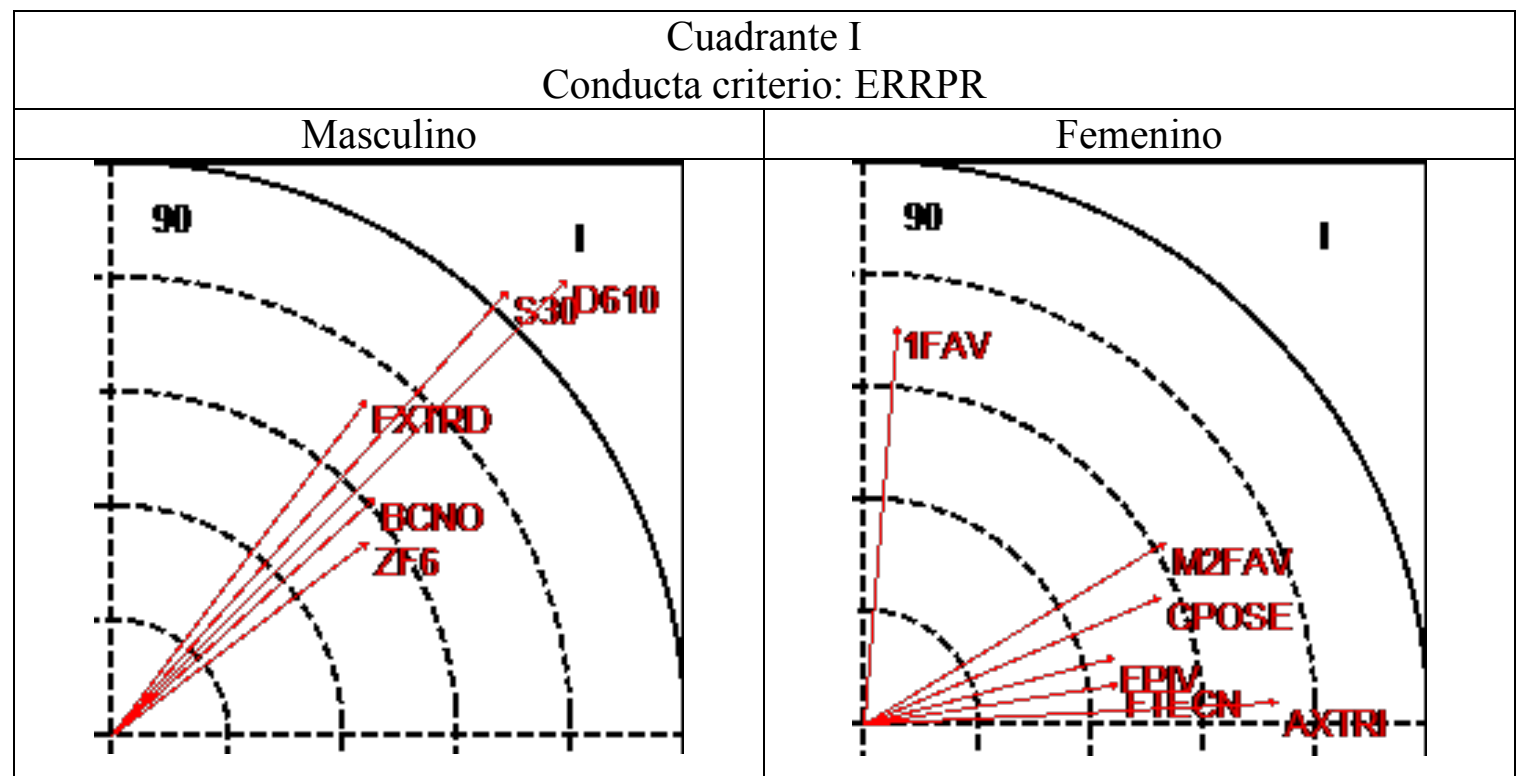

Figura 1. Representación gráfica de los vectores en el primer cuadrante en las dos categorías tomando como conducta criterio ERRPR.

Igualmente, cuando la conducta criterio es el FLY (recepcionando y lanzando el jugador el móvil en el aire), no se encuentran coincidencias en el tipo de conductas de apareo entre las categorías masculinas y femeninas.

En el estudio del doble portero como jugador que finaliza los ataques como conducta focal (FPETO), se encuentran tres conductas de apareo que se repiten en las dos categorías. Éstas son: más de dos goles a favor (M2FAV) en relación al marcador al inicio del ataque del equipo observado, el sistema defensivo contrario 3:0 (S30) y la zona de finalización 7 (ZF7: zona lateral izquierda dentro del área contraria). Además, también se encuentran otras conductas de apareo que difieren de la categoría masculina con la femenina.

Cuando se centra el estudio en las relaciones que se establecen con el pivote como jugador que finaliza (FPIV) como conducta criterio no se observan conductas de apareo que coincidan entre la categoría masculina y femenina.

El lanzamiento en giro es otra de las maneras de obtener valor doble al conseguir gol, por lo que esta conducta ha sido utilizada como focal para estudiar las posibles vinculaciones que se establecen con el resto de conductas. Los resultados muestran dos coincidencias en cuanto al número de conductas de apareo entre la categoría masculina y femenina: el marcador al inicio del ataque con más de dos puntos en contra (M2CON) y el propio lanzamiento en giro (GIRO). Como sucede con el resto de conductas criterio, se muestran conductas de apareo diferentes en cada categoría. La última conducta criterio estudiada ha sido el lanzamiento (LANZ), mayoritariamente realizado por el jugador que adopta el rol de doble portero. La única conducta de apareo coincidente entre ambas categorías está relacionada con el marcador al inicio del ataque; y es más de dos puntos a favor (M2FAV). El resto de conductas de apareo que se vinculan de manera significativa han sido cuatro en cada categoría, aunque éstas son diferentes. 
Morillo-Baro, J. P.; Reigal, R.; L.; Hernández-Mendo, A. (2015). Análisis del ataque posicional de balonmano playa masculino y femenino mediante coordenadas polares. RICYDE. Revista internacional de ciencias del deporte, 41(11), 226-244. http://dx.doi.org/10.5232/ricyde2015.04103

A continuación, en la tabla 5, se observa la vinculación significativa entre cada conducta focal y las conductas de apareo que se muestran en el cuadrante II, por lo que la conducta criterio se presenta como inhibitoria y las conductas de apareo como excitatorias.

Tabla 5. Relaciones significativas en el cuadrante II entre las conductas criterio y las conductas de apareo en las dos categorías.

\begin{tabular}{|c|c|c|c|c|c|c|}
\hline Cuadrante II & \multicolumn{3}{|c|}{ Masculino } & \multicolumn{3}{|c|}{ Femenino } \\
\hline $\begin{array}{l}\text { Conducta } \\
\text { criterio }\end{array}$ & $\begin{array}{l}\text { Conducta } \\
\text { de apareo }\end{array}$ & $\begin{array}{l}\text { Módulo } \\
\text { Vector }\end{array}$ & $\begin{array}{l}\text { Ángulo } \\
\text { transformado }\end{array}$ & $\begin{array}{l}\text { Conducta } \\
\text { de apareo }\end{array}$ & $\begin{array}{l}\text { Módulo } \\
\text { Vector }\end{array}$ & $\begin{array}{l}\text { Ángulo } \\
\text { transformado }\end{array}$ \\
\hline \multirow{4}{*}{ APETO } & $\mathrm{S} 21$ & 2.11 & 169.94 & GOL2R & 2.61 & 106.40 \\
\hline & ERLAN & 2.22 & 106.25 & 6MGSA & 2.02 & 111.41 \\
\hline & $6 \mathrm{MFA}$ & 1.98 & 143.35 & & & \\
\hline & LANZ & 2.15 & 93.89 & & & \\
\hline \multirow{4}{*}{ ERRPR } & ZF9 & 2.90 & 92.1 & MPATE & 2.70 & 167.77 \\
\hline & \multirow{3}{*}{\multicolumn{3}{|c|}{ 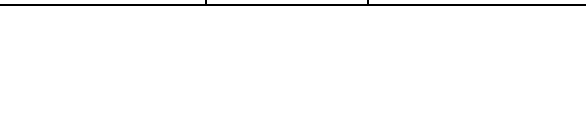 }} & APIV & 3.75 & 103.86 \\
\hline & & & & $6 \mathrm{M}$ & 2.04 & 114.25 \\
\hline & & & & 6MGSA & 3.01 & 96.24 \\
\hline FLY & FPETO & 2.20 & 118.47 & FCENT & 2.17 & 132.01 \\
\hline FPETO & & & & $\mathrm{M} 2 \mathrm{CON}$ & 2.64 & 148.47 \\
\hline \multirow{2}{*}{ FPIV } & D610 & 4.14 & 112.41 & M2FAV & 4.09 & 107.31 \\
\hline & & & & ACENT & 2.33 & 169.58 \\
\hline \multirow{2}{*}{ GIRO } & $2 \mathrm{CON}$ & 2.92 & 109.15 & $2 \mathrm{CON}$ & 2.36 & 142.99 \\
\hline & D1620 & 2.77 & 98.69 & S21 & 2.52 & 103.61 \\
\hline \multirow{2}{*}{ LANZ } & ZF7 & 3.31 & 100.47 & & & \\
\hline & $6 \mathrm{M}$ & 1.96 & 116.91 & & & \\
\hline
\end{tabular}

Valor de significación de las relaciones a nivel de $\mathrm{p}<0.05$

En general, en el cuadrante II se encuentra un número inferior de conductas de apareo que en el primer cuadrante; tanto en categoría masculina como femenina. En la primera, cuando la conducta criterio es APETO y se muestra como inhibitoria, se excitan cuatro categorías de apareo. En la femenina, sólo se vinculan significativamente dos conductas.

Cuando la conducta criterio es el error de pase o recepción, sólo se relaciona con la zona de finalización 9 (ZF9: zonal lateral derecha dentro del área contraria) en la categoría masculina; sin embargo, en la femenina se vincula con cuatro conductas.

Por otro lado, cuando la conducta criterio es el lanzamiento en FLY, sólo se vincula con una conducta para cada categoría, aunque no coinciden. En el caso de que la conducta criterio sea la finalización del doble portero, no se encuentran conductas de apareo en la categoría masculina y sólo una en la femenina. Pero si el jugador que finaliza es el pivote y se utiliza esta conducta como criterio, sí se encuentra una vinculación significativa en este segundo cuadrante en la categoría masculina, la relacionada con la duración del ataque entre 6 y 10 segundos. En la femenina se relaciona significativamente con el marcador con más de dos puntos a favor al inicio del ataque observado (M2FAV) y con las asistencias del central (ACENT).

Por último, la única coincidencia encontrada entre las categorías masculinas y femeninas en este segundo cuadrante se ha dado con el lanzamiento en giro como conducta criterio; y ha sido con el marcador de dos puntos en contra al inicio de la observación (2CON).

En la tabla 6 se observa la vinculación significativa entre cada conducta focal y las conductas de apareo que se muestran en ambas categorías en el cuadrante III; es decir, se presentan como mutuamente inhibitorias. 
Morillo-Baro, J. P.; Reigal, R.; L.; Hernández-Mendo, A. (2015). Análisis del ataque posicional de balonmano playa masculino y femenino mediante coordenadas polares. RICYDE. Revista internacional de ciencias del deporte, 41(11), 226-244. http://dx.doi.org/10.5232/ricyde2015.04103

Tabla 6. Relaciones significativas en el cuadrante III entre las conductas criterio y las conductas de apareo en las dos categorías.

\begin{tabular}{|c|c|c|c|c|c|c|}
\hline Cuadrante III & \multicolumn{3}{|c|}{ Masculino } & \multicolumn{3}{|c|}{ Femenino } \\
\hline $\begin{array}{l}\text { Conducta } \\
\text { criterio }\end{array}$ & $\begin{array}{l}\text { Conducta } \\
\text { de apareo }\end{array}$ & $\begin{array}{l}\text { Módulo } \\
\text { Vector }\end{array}$ & $\begin{array}{c}\text { Ángulo } \\
\text { transformado }\end{array}$ & $\begin{array}{l}\text { Conducta } \\
\text { de apareo }\end{array}$ & $\begin{array}{l}\text { Módulo } \\
\text { Vector }\end{array}$ & $\begin{array}{c}\text { Ángulo } \\
\text { transformado }\end{array}$ \\
\hline \multirow{9}{*}{ APETO } & $1 \mathrm{CON}$ & 2.47 & 189.46 & M2FAV & 3.36 & 187.94 \\
\hline & 1FAV & 2.19 & 245.17 & S30 & 3.56 & 237.37 \\
\hline & ACENT & 6.03 & 222.60 & APIV & 1.98 & 233.65 \\
\hline & ZF8 & 3.94 & 237.07 & ZF6 & 2.62 & 239.09 \\
\hline & FXTRI & 2.36 & 236.09 & GOL1 & 2.21 & 202.21 \\
\hline & FCENT & 2.65 & 226.03 & ERRPR & 2.12 & 205.36 \\
\hline & AXTRD & 2.08 & 250.44 & CPOSE & 2.86 & 208.26 \\
\hline & GOL1 & 3.66 & 265.35 & FXTRD & 2.32 & 242.01 \\
\hline & & & & D610 & 2.65 & 210.82 \\
\hline \multirow{5}{*}{ ERRPR } & S21 & 3.09 & 227.48 & $\mathrm{M} 2 \mathrm{CON}$ & 3.17 & 259.16 \\
\hline & D1115 & 4.31 & 223.33 & APETO & 2.12 & 244.64 \\
\hline & & & & FLY & 2.26 & 223.80 \\
\hline & & & & ERLAN & 2.58 & 224.16 \\
\hline & & & & D1620 & 2.24 & 204.10 \\
\hline \multirow{7}{*}{ FLY } & $1 \mathrm{CON}$ & 2.21 & 182.34 & $\mathrm{M} 2 \mathrm{CON}$ & 3.99 & 195.93 \\
\hline & BCSI & 2.04 & 197.95 & AXTRD & 2.25 & 214.60 \\
\hline & ZF6 & 3.84 & 211.94 & ZF6 & 3.69 & 245.05 \\
\hline & FXTRD & 2.72 & 235.50 & ERRPR & 2.26 & 226.20 \\
\hline & GIRO & 3.7 & 237.79 & FXTRD & 3.19 & 244.06 \\
\hline & D1115 & 2.58 & 245.76 & 6MGSA & 2.39 & 225 \\
\hline & D2630 & 3.27 & 261.38 & & & \\
\hline \multirow{6}{*}{ FPETO } & S21 & 2.86 & 255.57 & $1 \mathrm{FAV}$ & 2.58 & 254.30 \\
\hline & ACENT & 1.96 & 187.33 & ZF6 & 2.84 & 188.24 \\
\hline & LANZ & 2.29 & 217.06 & BCSI & 2.41 & 247.69 \\
\hline & & & & FXTRD & 3.67 & 198.53 \\
\hline & & & & GIRO & 3.68 & 231.01 \\
\hline & & & & D1620 & 2.63 & 211.26 \\
\hline \multirow{9}{*}{ FPIV } & $\mathrm{M} 2 \mathrm{CON}$ & 3.72 & 182.60 & $\mathrm{M} 2 \mathrm{CON}$ & 4.56 & 208.66 \\
\hline & MPATE & 2.49 & 195.69 & BCNO & 2.14 & 258.62 \\
\hline & $2 \mathrm{CON}$ & 2.78 & 257.63 & ZF6 & 3.43 & 260.64 \\
\hline & $\mathrm{BCNO}$ & 2.07 & 233.46 & & & \\
\hline & CPOSE & 2.28 & 219.85 & & & \\
\hline & FXTRD & 2.13 & 247.37 & & & \\
\hline & $6 \mathrm{M}$ & 2.45 & 256.22 & & & \\
\hline & 6MGOL & 2.29 & 248.19 & & & \\
\hline & D2630 & 2.12 & 266.70 & & & \\
\hline \multirow{9}{*}{ GIRO } & 1FAV & 2.09 & 220.83 & $1 \mathrm{CON}$ & 2.43 & 204.68 \\
\hline & M2FAV & 5.71 & 266.28 & M2FAV & 4.57 & 230.92 \\
\hline & S30 & 2.85 & 226.02 & ZF5 & 3.38 & 235.69 \\
\hline & ZF9 & 3.14 & 202 & FPETO & 3.68 & 218.99 \\
\hline & FLY & 3.70 & 212.21 & BCMED & 3.90 & 230.88 \\
\hline & D05 & 2.93 & 202.22 & LANZ & 3.71 & 219.31 \\
\hline & D610 & 4.29 & 239.66 & CPOSE & 3.47 & 229.48 \\
\hline & & & & FTECN & 3.09 & 243.84 \\
\hline & & & & D610 & 2.16 & 230.28 \\
\hline \multirow[t]{7}{*}{ LANZ } & $\mathrm{M} 2 \mathrm{CON}$ & 5.62 & 222.33 & 1FAV & 2.88 & 228.27 \\
\hline & FPETO & 2.29 & 232.94 & ZF6 & 3.08 & 190.41 \\
\hline & DM30 & 2.24 & 191.46 & BCSI & 3.15 & 233.66 \\
\hline & S21 & 4.35 & 269.99 & FXTRD & 3.37 & 195.19 \\
\hline & & & & GIRO & 3.71 & 230.69 \\
\hline & & & & 1SUP & 2.10 & 213.66 \\
\hline & & & & $\mathrm{S} 20$ & 2.10 & 213.66 \\
\hline
\end{tabular}


Morillo-Baro, J. P.; Reigal, R.; L.; Hernández-Mendo, A. (2015). Análisis del ataque posicional de balonmano playa masculino y femenino mediante coordenadas polares. RICYDE. Revista internacional de ciencias del deporte, 41(11), 226-244. http://dx.doi.org/10.5232/ricyde2015.04103

\begin{tabular}{|c|c|c|c|c|c|c|}
\hline Cuadrante III & \multicolumn{3}{|c|}{ Masculino } & \multicolumn{3}{c|}{ Femenino } \\
\hline $\begin{array}{c}\text { Conducta } \\
\text { criterio }\end{array}$ & $\begin{array}{c}\text { Conducta } \\
\text { de apareo }\end{array}$ & $\begin{array}{c}\text { Módulo } \\
\text { Vector }\end{array}$ & $\begin{array}{c}\text { Ángulo } \\
\text { transformado }\end{array}$ & $\begin{array}{c}\text { Conducta } \\
\text { de apareo }\end{array}$ & $\begin{array}{c}\text { Módulo } \\
\text { Vector }\end{array}$ & $\begin{array}{c}\text { Ángulo } \\
\text { transformado }\end{array}$ \\
\hline & \multicolumn{3}{|c|}{} & D1620 & 2.69 & 193.54 \\
\hline
\end{tabular}

Valor de significación de las relaciones a nivel de $\mathrm{p}<0.05$

Varias son las conductas de apareo que se relacionan con las categorías masculina y femenina cuando la categoría focal es la asistencia del peto, pero sólo una de ellas coincide en ambas; esta es el gol de valor simple (GOL1). El resto de conductas de apareo son diferentes y muestran la diferente orientación de las acciones en cada categoría.

Cuando la conducta criterio es el error de pase o recepción, se encuentran más conductas de apareo en la categoría femenina (5) que en la masculina (2) en este tercer cuadrante.

En una de las conductas criterio que describe uno de los modos de finalización, como es el lanzamiento en flight, su vinculación con el resto de conductas desde el punto de vista de conducta criterio vuelve a ser diferente en la categoría masculina y femenina; aunque se encuentran dos conductas de apareo que se repiten.

Al estudiar las relaciones que se establecen en la finalización del ataque desde el punto de vista de la conducta criterio FPETO (finaliza el doble portero), en la figura 2 se observan de manera gráfica los resultados observados en la tabla 6, y que muestran grandes diferencias entre la categoría masculina y femenina. Se destaca que el número de conductas de apareo se duplica en la femenina y que no coinciden ninguna.

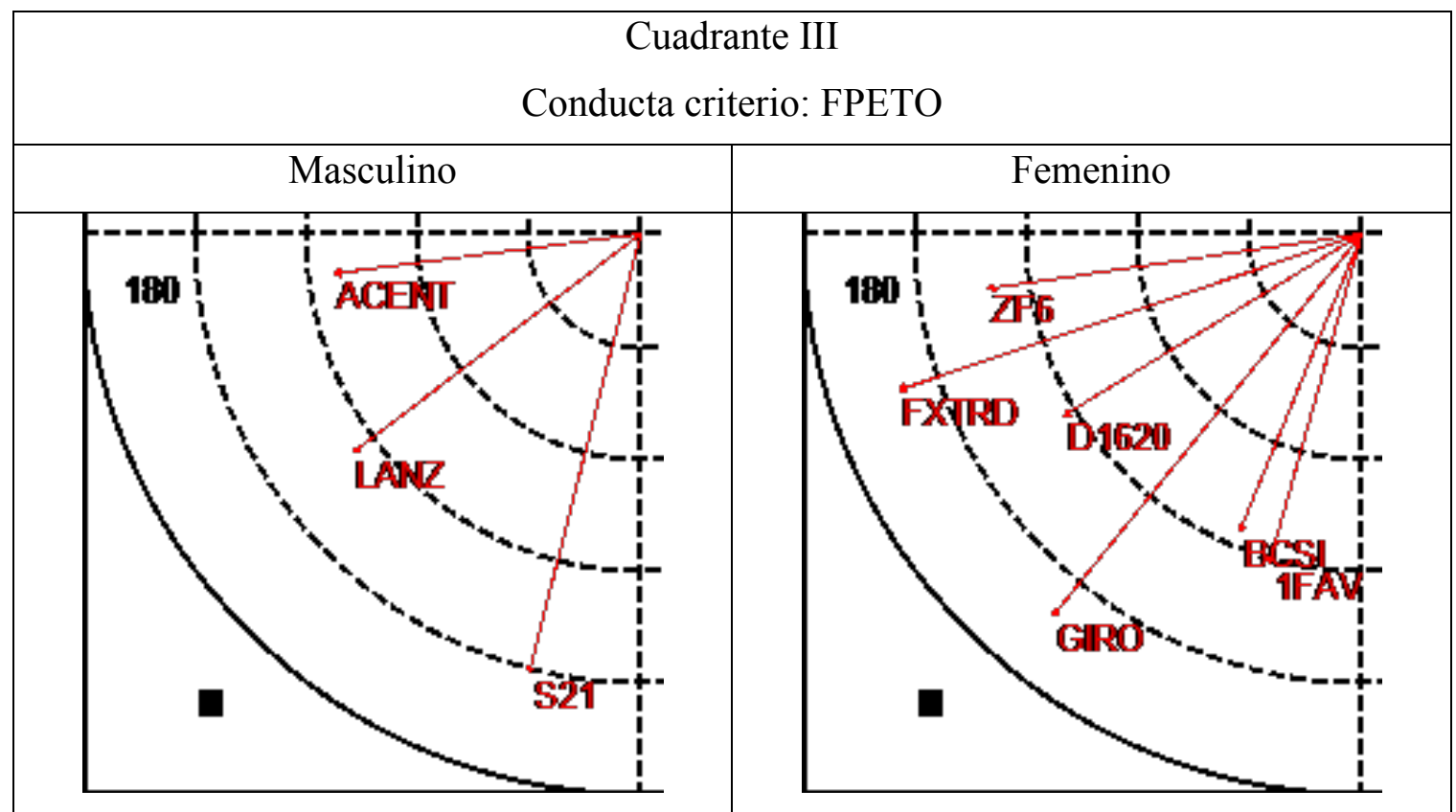

Figura 2. Representación gráfica de los vectores en el tercer cuadrante en las dos categorías tomando como conducta criterio FPETO.

Continuando con el análisis utilizando una conducta focal desde el punto de vista del puesto específico que finaliza, en este caso el pivote, en los resultados se observan también grandes diferencias entre las dos categorías. Ahora el número de conductas de apareo se triplica en la categoría masculina, pero sí coinciden dos de ellas.

La mutua inhibición entre la conducta criterio lanzamiento en giro y las conductas de apareo sólo coinciden en dos al comparar los resultados de la categoría masculina con la femenina: el marcador de más de dos puntos a favor al inicio del ataque y la duración de los ataques entre 
Morillo-Baro, J. P.; Reigal, R.; L.; Hernández-Mendo, A. (2015). Análisis del ataque posicional de balonmano playa masculino y femenino mediante coordenadas polares. RICYDE. Revista internacional de ciencias del deporte, 41(11), 226-244. http://dx.doi.org/10.5232/ricyde2015.04103

6 y 10 segundos. El resto de conductas de apareo en cada población son diferentes al otro grupo. El número de conductas de apareo en categoría femenina duplica a la masculina (8 frente a 4) cuando se finaliza con un lanzamiento del doble portero y de nuevo se repite la no coincidencia de estas conductas entre las dos poblaciones estudiadas.

Por último, en la tabla 7 se observa la vinculación significativa entre cada conducta focal y las conductas de apareo que se muestran en el cuadrante IV, por lo que la conducta criterio se presenta como excitatoria y las conductas de apareo como inhibitorias.

Tabla 7. Relaciones significativas en el cuadrante IV entre las conductas criterio y las conductas de apareo en las dos categorías.

\begin{tabular}{|c|c|c|c|c|c|c|}
\hline \multirow{2}{*}{$\begin{array}{l}\text { Cuadrante IV } \\
\begin{array}{l}\text { Conducta } \\
\text { criterio }\end{array} \\
\end{array}$} & \multicolumn{3}{|c|}{ Masculino } & \multicolumn{3}{|c|}{ Femenino } \\
\hline & $\begin{array}{l}\text { Conducta } \\
\text { de apareo }\end{array}$ & $\begin{array}{l}\text { Módulo } \\
\text { Vector }\end{array}$ & $\begin{array}{l}\text { Ángulo } \\
\text { transformado }\end{array}$ & $\begin{array}{l}\text { Conducta } \\
\text { de apareo }\end{array}$ & $\begin{array}{l}\text { Módulo } \\
\text { Vector }\end{array}$ & $\begin{array}{l}\text { Ángulo } \\
\text { transformado }\end{array}$ \\
\hline \multirow{5}{*}{ APETO } & $2 \mathrm{FAV}$ & 1.97 & 307.57 & ACENT & 2.06 & 275.37 \\
\hline & SPRES & 2.32 & 275.79 & & & \\
\hline & CPOSE & 2.19 & 322.02 & & & \\
\hline & D05 & 2.38 & 270.20 & & & \\
\hline & D2125 & 2.07 & 332.10 & & & \\
\hline \multirow{2}{*}{ ERRPR } & $\mathrm{M} 2 \mathrm{CON}$ & 2.03 & 285.33 & FCENT & 2.14 & 299.55 \\
\hline & D2630 & 1.98 & 327.20 & GOL2R & 2.31 & 332.65 \\
\hline \multirow{3}{*}{ FLY } & IGUAL & 2.34 & 331.89 & $2 \mathrm{CON}$ & 4.77 & 329.11 \\
\hline & S30 & 2.38 & 274.81 & & & \\
\hline & D1620 & 2.09 & 275.65 & & & \\
\hline \multirow{4}{*}{ FPETO } & 2FAV & 2.12 & 275.56 & ZF4 & 2.30 & 288.98 \\
\hline & MPATE & 2.06 & 347.73 & & & \\
\hline & $1 \mathrm{INF}$ & 2.11 & 285.56 & & & \\
\hline & FLY & 2.20 & 331.53 & & & \\
\hline \multirow{2}{*}{ FPIV } & D1115 & 2.10 & 317.83 & $2 \mathrm{CON}$ & 2.96 & 311.65 \\
\hline & D1620 & 3.21 & 270.48 & FXTRD & 2.43 & 285.38 \\
\hline \multirow{2}{*}{ GIRO } & DM30 & 2.19 & 346.91 & $\mathrm{~S} 30$ & 2.79 & 273.87 \\
\hline & & & & APIV & 2.35 & 272 \\
\hline \multirow[t]{2}{*}{ LANZ } & MPATE & 2.72 & 336.73 & ZF4 & 2.88 & 278.11 \\
\hline & $1 \mathrm{CON}$ & 2.38 & 277.24 & & & \\
\hline
\end{tabular}


Morillo-Baro, J. P.; Reigal, R.; L.; Hernández-Mendo, A. (2015). Análisis del ataque posicional de balonmano playa masculino y femenino mediante coordenadas polares. RICYDE. Revista internacional de ciencias del deporte, 41(11), 226-244. http://dx.doi.org/10.5232/ricyde2015.04103

\begin{tabular}{|l|l|l|l|l|l|l|}
\hline \multirow{2}{*}{ Cuadrante IV } & \multicolumn{3}{|c|}{ Masculino } & \multicolumn{3}{c|}{ Femenino } \\
\hline $\begin{array}{l}\text { Conducta } \\
\text { criterio }\end{array}$ & $\begin{array}{l}\text { Conducta } \\
\text { de apareo }\end{array}$ & $\begin{array}{l}\text { Módulo } \\
\text { Vector }\end{array}$ & $\begin{array}{l}\text { Ángulo } \\
\text { transformado }\end{array}$ & $\begin{array}{l}\text { Conducta } \\
\text { de apareo }\end{array}$ & $\begin{array}{l}\text { Módulo } \\
\text { Vector }\end{array}$ & $\begin{array}{l}\text { Ángulo } \\
\text { transformado }\end{array}$ \\
\hline & 2FAV & 2.64 & 310.13 & & \\
\cline { 2 - 5 } & APETO & 2.15 & 356.11 & & \\
\cline { 2 - 4 } & ZF9 & 2.67 & 298.99 & & \\
\hline
\end{tabular}

Valor de significación de las relaciones a nivel de $\mathrm{p}<0.05$

En primer lugar, en este cuarto cuadrante y al comparar los resultados entre las dos categorías, se observa una total discrepancia entre las conductas de apareo en las dos categorías.

Siguiendo el mismo esquema que en el análisis de los resultados de los cuadrantes anteriores, se destaca que en el estudio de la conducta criterio de las asistencias que realiza el jugador que adquiere el rol de doble portero, en la categoría femenina sólo se vincula con las asistencias del central; sin embargo, en la masculina se relaciona con cuatro conductas de apareo. Los errores de pase y recepción se relacionan, en la categoría masculina, con el marcador de más de dos puntos en contra y con la duración de los ataques de entre 26 y 30 segundos; sin embargo, en la femenina se vincula con la finalización de la jugadora central y con el gol de valor doble obtenido tras un rebote.

La conducta criterio finaliza el doble portero sólo se vincula con una conducta de apareo en la categoría femenina, en este caso con la zona de finalización lateral izquierda fuera del área. No sucede lo mismo en la categoría masculina, donde se vincula con cuatro conductas de apareo. De nuevo no coinciden las dos conductas de apareo que se presentan entre las categorías estudiadas al utilizar la conducta criterio finaliza el pivote.

En el caso de que el modo de finalización mediante un lanzamiento en giro sea la conducta criterio, los resultados muestran una sola conducta de apareo en la categoría masculina, la relacionada con la duración de los ataques con más de 30 segundos. En la otra categoría se relaciona con el sistema defensivo contrario 3:0 y las asistencias de la pivote.

Por último, la conducta criterio lanzamiento del doble portero muestra una relación significativa con la zona de finalización lateral izquierda fuera del área en la categoría femenina. En la masculina, además de con otras cuatro conductas, la zona de finalización con que se vincula es la lateral derecha dentro del área contraria.

\section{Discusión}

El objetivo de este estudio fue identificar mediante análisis de coordenadas polares las relaciones y asociaciones entre las diferentes conductas que suceden en el ataque posicional del balonmano playa. La investigación se ha desarrollado sobre dos grupos de población diferentes, la categoría masculina y la femenina, con la intención de revelar si los flujos de conductas en cada fase de ataque son coincidentes. Además, el procedimiento de la investigación mediante el software de observación Hoisan (Hernández-Mendo, López-López, Castellano, Morales-Sánchez, y Pastrana, 2012; Hernández-Mendo y col., 2014) ha permitido salvar dificultades como la no disposición de un programa informático que integrase análisis secuenciales y de coordenadas polares, los cálculos matemáticos y su representación vectorial conjuntamente (Perea y col., 2012).

El estudio de las conductas de juego en balonmano mediante el análisis de coordenadas polares (González y col., 2013; Prudente y col., 2010) ha permitido comprender mejor la 
Morillo-Baro, J. P.; Reigal, R.; L.; Hernández-Mendo, A. (2015). Análisis del ataque posicional de balonmano playa masculino y femenino mediante coordenadas polares. RICYDE. Revista internacional de ciencias del deporte, 41(11), 226-244. http://dx.doi.org/10.5232/ricyde2015.04103

realidad de este deporte; el cual no es comparable al balonmano playa y del que no existen estudios similares. Los resultados de esta pionera investigación han mostrado diferencias entre las conductas de apareo en la categoría masculina y femenina; destacando que el ataque posicional en la femenina se orienta hacia zonas de finalización izquierdas ante un sistema defensivo abierto y depende más de la jugadora que adquiere el rol de doble portera que en la categoría masculina, donde las responsabilidades están más repartidas y el ataque se dirige hacia la banda derecha ante un sistema defensivo cerrado. Además, el lanzamiento en giro se ha mostrado como el principal recurso ofensivo en ambas categorías.

Profundizando en el análisis e interpretación de los resultados, las vinculaciones de mutua excitación que se establecen en el primer cuadrante entre las conductas focales seleccionadas y las conductas de apareo resultantes dirigen el ataque en balonmano playa en direcciones diferentes dependiendo de si la categoría es masculina o femenina. En los chicos, cuando la conducta criterio es la asistencia del doble portero se asocia significativamente a un sistema defensivo cerrado y a la finalización por la zona del lateral derecho en flight; sin embargo, en las chicas se asocia a sistemas defensivos abiertos, a la finalización del lateral izquierdo en su zona en flight y a los errores de lanzamiento. Se entiende que estas diferencias en los sistemas defensivos se deben a la necesidad que presenta el juego femenino de limitar las acciones de esta jugadora, ya que en esta categoría su participación en la toma de decisiones y finalización es más determinante para el resultado que en la masculina, donde estas cualidades están más repartidas entre todos los jugadores. Además, la finalización del ataque mediante un lanzamiento en flight se orienta hacia zonas contrarias debido a la lateralidad de las jugadoras.

Los errores de pase o recepción se vinculan, entre otras conductas y en la categoría masculina, con el sistema defensivo cerrado y con el lateral derecho cometiéndolos en su zona habitual. Sin embargo, en la femenina se orienta hacia el pivote mediante falta técnica principalmente. Se entiende que en la femenina el pivote tiene más opciones de participar debido a que se enfrenta a un sistema defensivo abierto. La orientación del entrenamiento en el puesto específico del pivote debe atender a esta necesidad trabajando la toma de decisiones y no sólo su faceta finalizadora.

En el análisis de los resultados de la finalización del doble portero, destacar que las coincidencias entre las categorías se establecen en el marcador favorable ante un sistema defensivo contrario cerrado, ya que se le permite entrar en su zona eficaz de lanzamiento a distancia. Y los lanzamientos en giro, que permiten la obtención de valor doble, se muestran como el más utilizado por los equipos de ambas categorías para buscar la seguridad en el éxito de la finalización.

Las diferencias entre el flujo de conductas en la fase de ataque en balonmano playa masculino y femenino no sólo se centran en el primer cuadrante, sino que se manifiestan en los cuatro. En el segundo destaca que se encuentra un número total de conductas de apareo inferior al primero. Además, en la categoría masculina es muy reseñable que cuando la conducta criterio que define las asistencias del doble portero se inhibe, se vincula significativamente con una excitación de la conducta de apareo que hace mención a un sistema defensivo contrario abierto con más profundidad en la zona central, que dificulta esta faceta creativa del jugador. También se excita la faceta finalizadora de este jugador mediante los lanzamientos, por lo que la participación de este jugador es constante, ya que si no asiste se potencia su capacidad finalizadora.

Igualmente destacable, aunque sólo en la categoría femenina, es que cuando la conducta focal inhibida es la finalización por parte del doble portero se excita la conducta de apareo del marcador al inicio del ataque observado con más de dos puntos en contra; aspecto que revela la importancia y dependencia de esta jugadora en el desarrollo del juego ofensivo femenino y 
Morillo-Baro, J. P.; Reigal, R.; L.; Hernández-Mendo, A. (2015). Análisis del ataque posicional de balonmano playa masculino y femenino mediante coordenadas polares. RICYDE. Revista internacional de ciencias del deporte, 41(11), 226-244. http://dx.doi.org/10.5232/ricyde2015.04103

que nos plantea la necesidad de distribuir las responsabilidades, tanto creativas como finalizadoras, entre todos los puestos específicos.

En el tercer cuadrante la conducta criterio y las conductas de apareo se muestran como mutuamente inhibitorias. Aunque se encuentran ligeras coincidencias entre las conductas de apareo en las dos categorías, en la mayoría de las ocasiones no es así. Destaca, en ambas categorías, que cuando se inhibe la conducta focal que describe los lanzamientos en flight, se inhibe la finalización del lateral derecho. Datos que muestran la importancia de este modo de finalización para este puesto específico y la dependencia de un compañero que le asista; al tiempo que manifiesta la carencia de la finalización mediante lanzamientos en giro y se postula como una propuesta de mejora en los entrenamientos.

La conducta criterio GIRO muestra dos coincidencias entre las dos categorías, destacando el marcador de más de dos puntos a favor al inicio del ataque observado. Este tipo de lanzamientos es el principal recurso ofensivo de los equipos, por lo que inhibir su aparición se refleja en el marcador de manera significativa. Además, resaltar por otro lado que los lanzamientos como conducta focal se muestran, en la categoría masculina, como mutuamente inhibitorios con la finalización del doble portero. Él es el único jugador al que se le puntúan doblemente, por lo que su utilización por cualquier otro jugador se reduce a las situaciones de necesidad de sumar goles de valor simple.

Finalmente, la relaciones que se establecen entre la conducta criterio y las conductas de apareo en el cuarto cuadrante se muestran como excitatoria de la primera e inhibitorias de las segundas. Volviendo a manifestar los resultados de los análisis las diferencias entre las dos categorías al no coincidir ninguna de las conductas de apareo. Muestra de ello es que, en la categoría masculina, la conducta criterio FLY se vincula inhibiendo el sistema defensivo cerrado 3:0, caracterizado por presentar mayor anchura y dificultar las acciones de los jugadores laterales. Sin embargo en la categoría femenina, la conducta criterio que inhibe este sistema defensivo es el lanzamiento en giro.

Igualmente en la categoría masculina, es reseñable que la finalización del pivote y el lanzamiento en giro como conductas criterio inhiben la duración larga del ataque. Efectivamente, la condición de superioridad numérica del equipo que ataca permite encontrar soluciones con rapidez, acciones que se acentúan si las realiza el pivote debido a su posición; o mediante lanzamientos en giro, ya que estos últimos son acciones técnicas que precisan escasa o nula colaboración de un compañero.

Las diferencias en el flujo de conductas del ataque posicional entre las categorías masculina y femenina en el deporte de balonmano playa han sido descritas con la utilización del análisis de coordenadas polares. Técnica que se ha mostrado como una herramienta útil para comprobar estas asociaciones de conductas y mostrar la orientación del entrenamiento en cada una de ellas ante la competición. y como prospectiva, se requiere seguir profundizando en este tipo de análisis en las próximas competiciones. 
Morillo-Baro, J. P.; Reigal, R.; L.; Hernández-Mendo, A. (2015). Análisis del ataque posicional de balonmano playa masculino y femenino mediante coordenadas polares. RICYDE. Revista internacional de ciencias del deporte, 41(11), 226-244. http://dx.doi.org/10.5232/ricyde2015.04103

\section{Referencias}

Anguera, M.T. (1990). Metodología observacional. En J. Arnau, M. T. Anguera, y J. Gómez-Benito (Eds.), Metodología de la investigación en ciencias del comportamiento (pp. 125-236). Murcia: Universidad de Murcia.

Anguera, M.T., y Hernández-Mendo, A. (2013). La metodología observacional en el ámbito del deporte. E-balonmano.com: Revista de Ciencias del Deporte 9(3), 135160.

Anguera, M.T., y Hernández-Mendo, A. (2014). Metodología observacional y psicología del deporte: Estado de la cuestión. Revista de Psicología del Deporte, 23(1), 103109.

Anguera, M. T.; Blanco, A., y Losada, J. L. (1997). Aportaciones de la técnica de coordenadas polares en diseños mixtos. En IV Simposio de metodología de las ciencias del comportamiento (p. 583). Murcia: Universidad de Murcia.

Anguera, M.T.; Blanco, A.; Hernández-Mendo, A., y Losada, J.L. (2011). Diseños observacionales: ajuste y aplicación en psicología del deporte. Cuadernos de Psicología del Deporte, 11(2), 63-76.

Blanco, A.; Castellano, J., y Hernández-Mendo, A. (2000). Generalizabilidad de las observaciones de la acción del juego en el fútbol. Psicothema, 12(2), 81-86.

Blanco-Villaseñor, A.; Castellano, J.; Hernández-Mendo, A.; Sánchez-López, C. R., y Usabiaga, O. (2014). Aplicación de la TG en el deporte para el estudio de la fiabilidad, validez y estimación de la muestra. Revista de Psicología del Deporte, 23(1), 131-137.

Carling, C.; Williams, A., \& Reilly, T. (2005). The handbook of soccer match analysis. Londres: Routledge.

http://dx.doi.org/10.4324/9780203448625

Casamichana, D.; Castellano, J.; Blanco-Villaseñor, A., y Usabiaga, O. (2012). Estudio de la percepción subjetiva del esfuerzo en tareas de entrenamiento en fútbol a través de la teoría de la generalizabilidad. Revista de Psicología del Deporte, 21 (1), 35-40.

Castellano, J., y Hernández-Mendo, A. (2003). El análisis de coordenadas polares para la estimación de relaciones en la interacción motriz en fútbol. Psicothema, 15(4), 569579. Recuperado el 23/10/2014, disponible en: http://www.psicothema.com/psicothema.asp?id=1109

Castellano, J.; Hernández-Mendo, A.; Morales-Sánchez, V., \& Anguera, M.T. (2007). Optimising a probabilistic model of the development of play in soccer. Quality and Quantity, 41(1), 93-104.

http://dx.doi.org/10.1007/s11135-005-3148-0

Garzón, B.; Lapresa, D.; Anguera, M.T., y Arana, J. (2011). Análisis observacional del lanzamiento de tiro libre en jugadores de baloncesto base. Psicothema, 23(4), 851857.

Gorospe, G., y Anguera, M.T. (2000). Modificación de la técnica clásica de coordenadas polares mediante un desarrollo distinto de la retrospectividad: aplicación al tenis. Psicothema, 12(2), 279-282.

González A.; Botejara, J.; Puñales, L.; Trejo, A., y Ruy, E. (2013). Análisis de la finalización del ataque en partidos igualados en balonmano de alto nivel mediante coordenadas polares. E-balonmano.com: Revista de Ciencias del Deporte 9(2), 7189. 
Morillo-Baro, J. P.; Reigal, R.; L.; Hernández-Mendo, A. (2015). Análisis del ataque posicional de balonmano playa masculino y femenino mediante coordenadas polares. RICYDE. Revista internacional de ciencias del deporte, 41(11), 226-244. http://dx.doi.org/10.5232/ricyde2015.04103

Hernández-Mendo, A., y Anguera, M.T. (1998). Análisis de coordenadas polares en el estudio de las diferencias individuales de la acción de juego. En: Sánchez, M. P., López Quiroga Estévez, M.A. (eds.). Perspectivas actuales en la investigación de las diferencias individuales, (pp. 84-88). Centro de Estudios Ramón Areces, Madrid.

Hernández-Mendo, A., y Anguera, M.T. (1999). Aportaciones de análisis de coordenadas polares a los deportes de equipo. En F. Guillén (Ed.), La Psicología del Deporte en España al final del milenio (pp. 169-175). Las Palmas: Universidad de Las Palmas de Gran Canaria.

Hernández-Mendo, A.; Castellano, J.; Camerino, O.; Jonsson, G.; Blanco-Villaseñor, A.; Lopes, A., y Anguera, M. T. (2014). Programas informáticos de registro, control de calidad del dato, y análisis de datos. Revista de Psicología del Deporte, 23(1), 111121.

Hernández-Mendo, A.; López López, J.A.; Castellano, J.; Morales-Sánchez, V., y Pastrana, J.L. (2012). Hoisan 1.2: Programa informático para uso en metodología observacional. Cuadernos de Psicología del Deporte, 12(1), 55-78. http://dx.doi.org/10.4321/S1578-84232012000100006

Lago, C., \& Anguera, M. T. (2002). Use of the polar coordinates technique to study interactions among professional soccer players. Revista Portuguesa de Ciências de Desporto, 2(4), 21-40.

Martín, R., y Lago, C. (2005). Deportes de equipo: comprender la complejidad para elevar el rendimiento. Barcelona: INDE Publicaciones.

Matsushigue, K.; Hartmann, K., \& Franchini, E. (2009). Taekwondo: physiological responses and match analysis. Journal of Strength and Conditioning Research, 23, 1112-1117.

http://dx.doi.org/10.1519/JSC.0b013e3181a3c597

Morillo, J. P. y Hernández-Mendo, A. (2015). Análisis de la calidad del dato de un instrumento para la observación del ataque en balonmano playa. Revista Iberoamericana de Psicología del Ejercicio y el Deporte, 10(1), 15 -22.

Perea, A. (2008). Análisis de las acciones colectivas en el fútbol de rendimiento. Tesis doctoral no publicada. Universidad del País Vasco.

Perea, A.; Castellano, J.; Alday, S., \& Hernández-Mendo, A. (2012). Analysis of behaviour in sports through Polar Coordinate Analysis with MATLAB. Quality and Quantity, 46(4), 1249-1260.

http://dx.doi.org/10.1007/s11135-011-9435-z

Prudente, J.; Garganta, J., \& Anguera, M. T. (2010). Methodological Approach to evaluate interactive behaviors in team games: An example in handball. En Proceedings of measuring behavior 2010. Eindhoven, Netherland. Recuperado el 23/10/2014, disponible en:

http://measuringbehavior.org/files/ProceedingsPDF(website)/Prudente_Symposium1.3.pdf

Robles, F.J., y Castellano, J. (2012). Comparación entre el juego ofensivo de la selección española de fútbol y sus rivales en la eurocopa'08 y mundial'10. Revista iberoamericana de psicología del ejercicio y el deporte, 7(2), 323-338.

Sarmento, H.; Marques, A.; Martins, J.; Anguera, M.T.; Campaniço, J., \& Leitão, J. (2011). Tactical analysis of the Barcelona counter-attack. British Journal of Sport Medicine, 45(15), A4.

http://dx.doi.org/10.1136/bjsports-2011-090606.12

Sousa, D.; Prudente, J.; Sequeira, P., y Hernandez-Mendo, A. (2014). Análise da qualidade dos dados de um instrumento para observação do 2 vs 2 no andebol. Revista Iberoamericana de Psicología del Ejercicio y el Deporte, 9(1), 173-190. 
Morillo-Baro, J. P.; Reigal, R.; L.; Hernández-Mendo, A. (2015). Análisis del ataque posicional de balonmano playa masculino y femenino mediante coordenadas polares. RICYDE. Revista internacional de ciencias del deporte, 41(11), 226-244. http://dx.doi.org/10.5232/ricyde2015.04103

Sackett, G.P. (1980). Lag Sequential Analysis as a data Reduction Technique in Social Interaction Research. En D.B. Sawin, R.C. Hawkins, L.O. Walker y J.H. Penticuff (Eds.), Exceptional infant. Phychosocial risks in infant-environment transactions (pp. 300-340). New York: Brunner/Mazel.

Santoyo, C., y Anguera, M.T. (1993). Evaluación ambiental: Integración de estrategias flexibles en situaciones naturales. En: M. Forns y M.T. Anguera (eds.), Aportaciones recientes a la evaluación psicológica (pp. 121-135). Barcelona: P.P.U.

Tornello, F.; Capranica, L.; Chiodo, S.; Minganti, C., \& Tessitore, A. (2013) Time-motion analysis of youth Olympic Taekwondo combats. Journal of Strength and Conditioning Research.27, 223-228.

http://dx.doi.org/10.1519/JSC.0b013e3182541edd

Tornello, F.; Capranica, L.; Minganti, C.; Chiodo, S.; Condello, G., \& Tessitore, A. (2014). Technical-Tactical Analysis of Youth Olympic Taekwondo Combat. Journal of Strength and Conditioning Research.28(4), 1151-1157. 